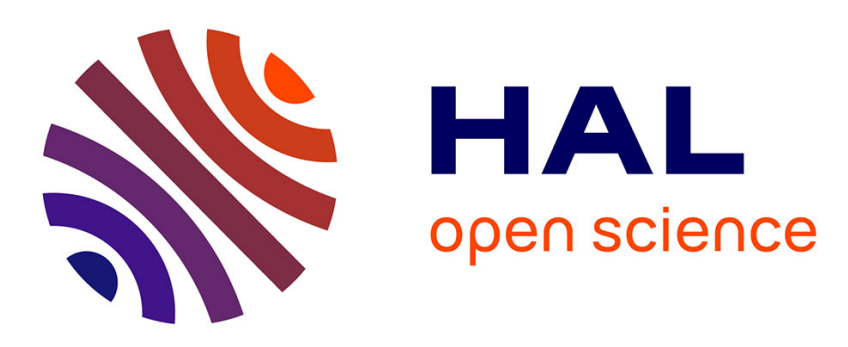

\title{
Closer: A New Design Principle for the Privileged Virtual Machine OS
}

Djob Mvondo, Boris Teabe, Alain Tchana, Daniel Hagimont, Noel de Palma

\section{To cite this version:}

Djob Mvondo, Boris Teabe, Alain Tchana, Daniel Hagimont, Noel de Palma. Closer: A New Design Principle for the Privileged Virtual Machine OS. IEEE 27th International Symposium on Modeling, Analysis, and Simulation of Computer and Telecommunication Systems (MASCOTS 2019), Oct 2019, Rennes, France. pp.49-60, 10.1109/MASCOTS.2019.00016 . hal-02333582

\section{HAL Id: hal-02333582 https://hal.science/hal-02333582}

Submitted on 25 Oct 2019

HAL is a multi-disciplinary open access archive for the deposit and dissemination of scientific research documents, whether they are published or not. The documents may come from teaching and research institutions in France or abroad, or from public or private research centers.
L'archive ouverte pluridisciplinaire HAL, est destinée au dépôt et à la diffusion de documents scientifiques de niveau recherche, publiés ou non, émanant des établissements d'enseignement et de recherche français ou étrangers, des laboratoires publics ou privés. 


\section{Closer: A new design principle for the privileged virtual machine OS}

\author{
Djob Mvondo \\ $L I G$ \\ Grenoble Alpes University \\ Grenoble, France \\ barbe-thystere.mvondo-djob@univ-grenoble-alpes.fr \\ Daniel Hagimont \\ IRIT \\ University of Toulouse \\ Toulouse, France \\ Daniel.Hagimont@enseeiht.fr
}

\author{
Boris Teabe \\ IRIT \\ University of Toulouse \\ Toulouse, France \\ boris.teabedjomgwe@enseeiht.fr \\ Alain Tchana \\ I3S \\ University of Nice \\ Nice, France \\ alain.tchana@univ-cotedazur.fr \\ Noel De Palma \\ $L I G$ \\ Grenoble Alpes University \\ Grenoble, France \\ noel.depalma@univ-grenoble-alpes.fr
}

\begin{abstract}
In most of today's virtualized systems (e.g., Xen), the hypervisor relies on a privileged virtual machine (pVM). The pVM accomplishes work both for the hypervisor (e.g., VM life cycle management) and for client VMs (I/O management). Usually, the pVM is based on a standard OS (Linux). This is source of performance unpredictability, low performance, resource waste, and vulnerabilities. This paper presents Closer, a principle for designing a suitable OS for the pVM. Closer consists in respectively scheduling and allocating pVM's tasks and memory as close to the involved client VM as possible. By revisiting Linux and Xen hypervisor, we present a functioning implementation of Closer. The evaluation results of our implementation show that Closer outperforms standard implementations.
\end{abstract}

Index Terms-Virtualization, Privileged VM, NUMA, ParaVirtualization

\section{INTRODUCTION}

The last decade has seen the widespread of virtualized environments, especially for the management of cloud computing infrastructures which implement the Infrastructure as a Service (IaaS) model. In a virtualized system, a low level kernel called the hypervisor runs directly atop the hardware. The hypervior's main goal is to provide the illusion of several virtual hardware to guest OSes (called virtual machines VMs). In order to keep its trusted computing base as smaller as possible, some of its functionalities (such as VM monitoring and life-cycle management tools) are provided by a privileged virtual machine (hereafter pVM), see Fig. 1.

Para-Virtualization (PV), introduced by Disco [1] and Xen [2], is a very popular virtualization approach which consists in making the guest OS aware of the fact that it runs in a virtualized environment. This approach has been proven to reduce virtualization overhead [3] and also mitigate the limitation of some virtualization hardware features (e.g. the inability to live migrate a VM with SR-IOV [4]-[6]). In this approach, the pVM plays a critical role because it hosts some device drivers (e.g. I/O) used by unprivileged VMs $(u V M s)$, making the pVM performing a lot of work on behalf of $\mathrm{uVMs}$. In this paper, we are interested in virtualization systems which rely on such a pVM.

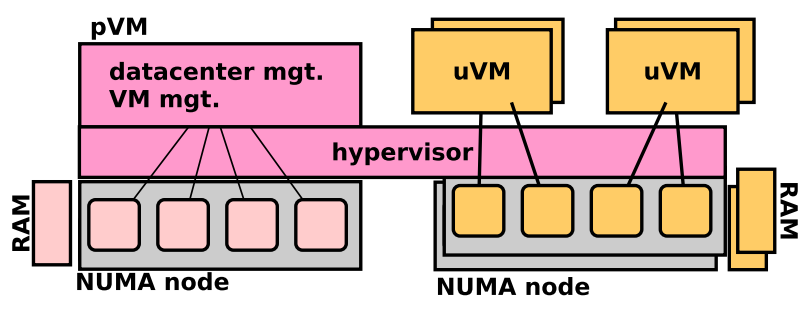

Fig. 1. Overall architecture of a virtualized system

The main issue with current pVMs is that they rely on standard OSes (e.g., Linux) whose resource manager does not consider pVM's particularities. The most important characteristic of the pVM is the correlation between pVM's tasks and uVM's activities. A significant part of $\mathrm{pVM}$ tasks are correlated with uVM's activities: (1) in terms of quantity, as the amount of resources required by the pVM depends on uVM's activities (especially I/O operations), and (2) in terms of location in a NUMA architecture, as resources (e.g. memory) allocated for one pVM's task are likely to be used by correlated uVM's activities.

A standard OS (running in a VM) manages resources for its applications and itself, but is not aware of resources managed in other VMs running on the same host. Therefore, the previous correlation between $\mathrm{pVM}$ and $\mathrm{uVMs}$ is not considered in a standard OS, thus leading to resource waste, low performance on NUMA architectures, performance unpredictability, and vulnerability to DoS attacks [7]-[9]. To take into account this correlation, the pVM should respect three rules: 


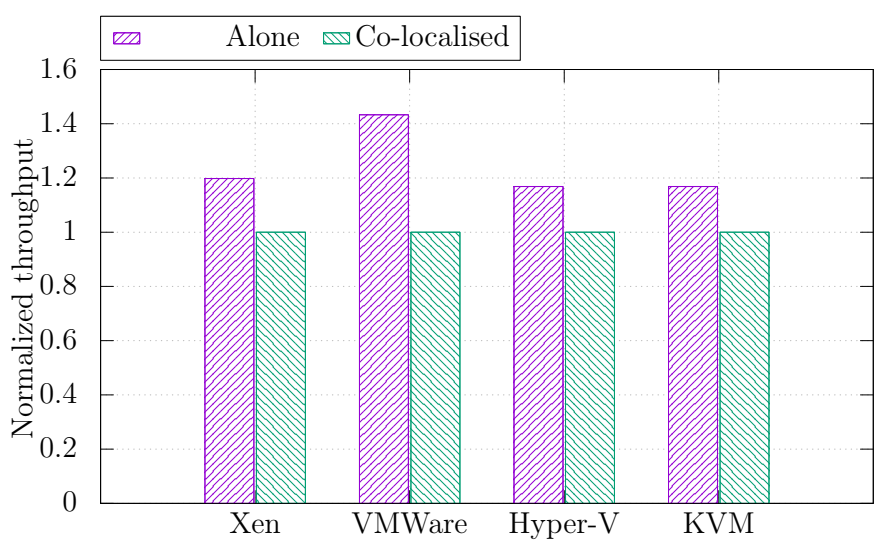

Fig. 2. In all existing hypervisors, the correlation of pVM's tasks with uVM's activities is not taken into account. This leads to several issues such as performance unpredictability, illustrated in this figure. Normalized performance of a website benchmark (wordpress) executed in two situations: alone and colocated with several other I/O intensive uVMs (normalized over the co-located situation). A predictable pVM management would have led to the same performance level regardless the colocated uVMs. The hypervisors versions are respectively Xen 4.9.1, VMWare ESXi 6.5, Hyper-V 2016 and Qemu-KVM 2.10.1

- on-demand. Resource allocation to the pVM should not be static. Resources should be allocated on demand according to $\mathrm{uVMs}$ activities. Without this rule, resources would be wasted if over-provisioned or they would be lacking, leading to performance unpredictability.

- pay-per-use. The resources allocated to a pVM task which is correlated with a uVM activity should be charged to the concerned uVM. Without this rule, the pVM would be vulnerable to DoS attacks from a VM executing on the same host, which could use most of the resources from the pVM.

- locality. The resources allocated to a pVM task which is correlated with a uVM activity should be located as close as possible (i.e. on the same NUMA node) to the uVM activity. This rule allows reducing remote memory accesses in the NUMA architecture, improving the uVM performance.

These rules are not currently respected by the pVM of the most popular virtualization systems.

In order to assess the significance of this issue, we evaluated the performance of a website (provided with wordpress [10]) executed within a VM in two situations. First, the VM runs alone on the machine. Second, the VM shares the machine with other VMs which perform intensive I/0 operations. We performed these experiments using popular hypervisors. In Fig. 2, we can observe that for all hypervisors, the performance of wordpress is unpredictable. We suspect (and we later demonstrate it with Xen) that this is caused by the saturation of the pVM, which size is fixed, thus not correlated with uVM's activities. In fact, by over-estimating the number od CPUs assigned to the pVM, we observed no performance variation.

In this paper, we propose Closer, a principle for implement- ing a suitable OS for the $\mathrm{pVM}$, Closer promotes the proximity (to $\mathrm{uVMs}$ ) and the utilization of uVM's resources. Following Closer, we propose a general architecture for the pVM. We revisit Linux thanks to this architecture, thus providing a ready to use OS dedicated to the pVM. We demonstrate the effectiveness using Xen, a popular open source virtualization system (used by Amazon). The final contribution of this paper is the instensive evaluation of our implementation usign both micro- and macro-benchamrks. The evaluation results show :

- No resource waste from the pVM, as the resources are allocated on-demand.

- Improvement of both VM life-cycle management tasks (creation, destruction, migration, etc.) and user's application performance in comparison with vanilla Linux (as the pVM OS) and Xen (as the hypervisor). We improve both VM destruction and migration time (by up to $33 \%$ ). Regarding user's application performance, we improve I/O intensive workloads (up to $36.50 \%$ for packet reception latency, $42.33 \%$ for packet emission latency, and $22 \%$ for disk operations).

- Pay-per-use and performance predictability enforcement, as pVM used resources are charged to the uVMs it works for.

The rest of the paper is organized as follows. Section II introduces key characteristics of virtualization systems where our works take place. Section III presents the motivations and an assessment of the problem. Section IV presents our contributions while Section $\mathrm{V}$ presents evaluation results. A review of the related work is presented in Section VI. The conclusion is drawn in Section VII.

\section{BACKGROUND}

Fig. 1 illustrates the general architecture of a virtualized environment. The hypervisor is the system layer which enforces resource sharing between virtual machines. For simplicity, maintainability and security purposes, a particular $\mathrm{VM}$ is used as an extension of the hypervisor. This VM is called the privileged VM $(p V M)$ while other VMs are called unpriviledged VMs $(u V M s)$. The pVM embeds uVM life-cycle administration tools (e.g., libxl in Xen, parent partition in Hyper-V) and data center administration applications (e.g novaCompute in OpenStack). In addition to these tasks, the pVM is also used in most deployments as a proxy for sharing and accessing I/O devices, see Fig. 3. In this case, the pVM embeds the driver enabling access to the hardware device, and a proxy (called backend) which relays incoming (from the driver to a $\mathrm{uVM}$ ) or outgoing (from a $\mathrm{uVM}$ to the driver) requests. Each $\mathrm{uVM}$ includes a fake driver (called frontend) allowing to send/receive requests to/from the uVM's backend. Therefore, each I/O operation performed by a uVM involves significant computations in the pVM. This architecture for I/O management is known as the split-driver model. It is widely used in datacenters for its very good performance and its high flexibility compared to hardwaresupported (HVM) solutions based on Single Root I/O Virtualization (SR-IOV) [11]. Indeed, SR-IOV allows a single 
device to be shared by multiple VMs while bypassing both the hypervisor and the $\mathrm{pVM}$ (the VM sends/receives packets directly to/from the device). However, SR-IOV is not widely adopted in datacenters as it comes with a strong limitation: VM migration should be disable [4]-[6], thus forbidding VM consolidation in the datacenter (which is critical for energy saving).
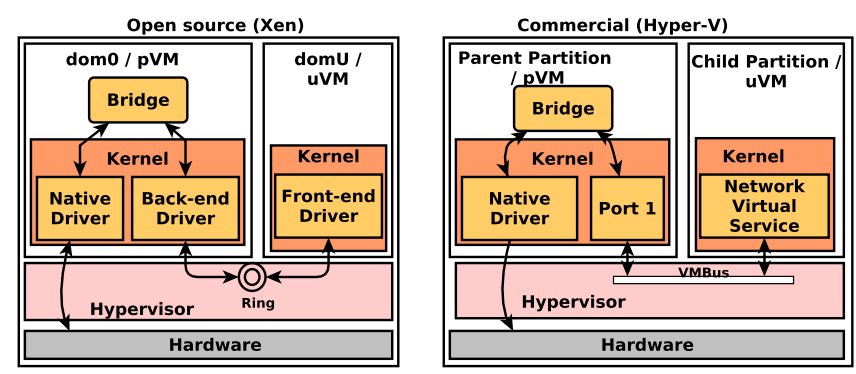

Fig. 3. Both open source (Xen) and commercial (Hyper-V) hypervisors implement the split-driver model. In the latter, the $\mathrm{pVM}$ provides access to $\mathrm{I} / \mathrm{O}$ devices on behalf of uVMs.

\section{Motivations}

Almost all previous research works have focused on resource allocation to $\mathrm{uVMs}$ since they host user applications. This paper shows that resource management for the pVM is a tricky task, and that it can have a significant impact on both administrative tasks and user applications. We are investigating this issue especially in NUMA architectures which are commonly used in today's datacenters.

A pVM resource management strategy must consider the three fundamental questions:

- $Q_{1}$ : how much resources (number of CPUs, amount of memory) should be allocated to the pVM?

- $Q_{2}$ : how to organize such an allocation (in terms of location) in a NUMA architecture?

- $Q_{3}$ : who (provider or user) these resources should be charged to?

The next sections discuss the current answers to those questions, and the related consequences.

\section{A. $\left(Q_{1}\right)$ pVM sizing}

Building on our experience (several collaboration with several datacenter operators ${ }^{1}$ ), we observed that static configuration is the commonly used strategy for $\mathrm{pVM}$ sizing, which means that the pVM is allocated a fixed amount of resources at startup. Moreover, virtualization system providers do not provide any recommendation regarding this issue. The only recommendation we found comes from Oracle [12] which proposes only a memory sizing strategy for the pVM based on the following formula: $p V M \_m e m=502+$ int (physical_mem $\times 0.0205)$. More generally, there isn't any

\footnotetext{
${ }^{1}$ One of our datacenter operator partner is in charge of up to 308 virtualized clusters around the world
}
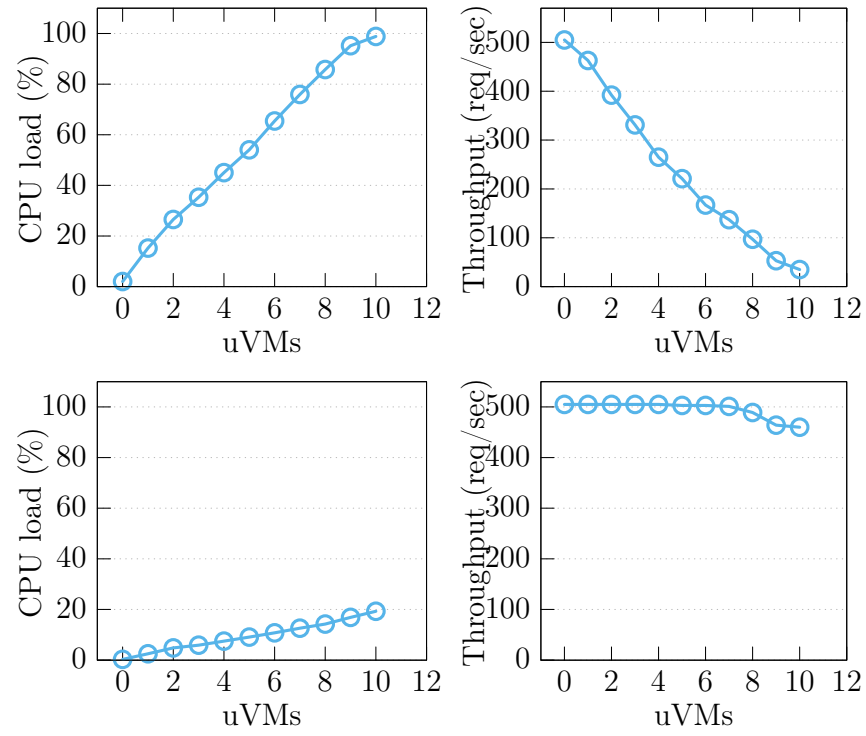

Fig. 4. A static resource allocation to the pVM can lead to its saturation, thus to performance unpredictability for user applications: (left) pVM's CPU load, (right) performance of the $\mathrm{uVM}$ which executes a (Wordpress) workload. The pVM has 2 CPUs for the top results and 12 CPUs for the bottom.
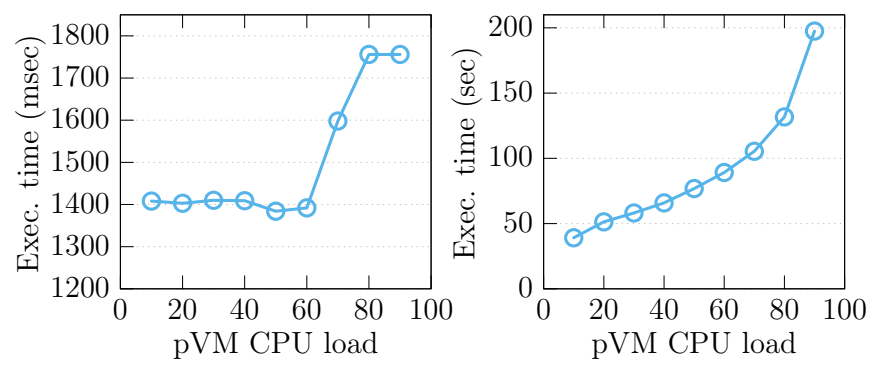

Fig. 5. A static resource allocation to the pVM may lead to its saturation, thus to variable uVM (left) creation and (right) migration time.

defined method to estimate the amount of resources required by the pVM to perform correctly.

The resources required by the $\mathrm{pVM}$ are not constant as they depend on $\mathrm{uVM}$ activities, therefore a static allocation cannot be the solution. In fact, the tasks executed by the $\mathrm{pVM}$ can be organized in two categories: tasks related to the management of the datacenter and tasks related to $\mathrm{uVM}$ I/O operations. The amount of resources required by tasks from the second category is fickle as it depends on uVM activities. A static allocation can lead to two situations, either the pVM's resources are insufficient or overbooked. These two situations can be harmful for both the cloud provider and user applications. We focus on the consequences of an insufficient resource provisioning to the $\mathrm{pVM}$ because it is obvious that over-provisioning causes resource waste as shown by many researchers [7]. The experimental environment used in this section is detailed in Section V-A.

A lack of resource in the pVM can make both applications executed in uVMs and administrative services executed in the 
pVM inefficient and unpredictable. The latter is known as one of the main issues in the cloud [8], [13], [14].

\section{Impact on user's applications}

Fig. 4 top right shows the performance of a uVM which hosts a web application (wordpress) on a physical machine where we vary the number of colocated $\mathrm{uVMs}$ which execute $\mathrm{I} / \mathrm{O}$ intensive workloads. In this experiment, the pVM is allocated two CPUs while each uVM is allocated one CPU. To prevent any contention (e.g. QPI link contention), all CPUs (from the pVM or uVMs) are allocated on the same NUMA socket. Fig. 4 top left presents the pVM CPU load during each experiment. The first observation is that the pVM load varies according to uVMs activities. This is due to the fact that the pVM embeds both the backends and the drivers responsible for accessing I/O devices (see Fig. 3). The second observation is that the web application's performance decreases when the pVM lacks CPU resources. Therefore, performance predictability is compromised.

One may wonder whether this unpredictability is effectively caused by lack of computation power for the pVM. Since all uVMs execute I/O intensive workloads, the I/O hardware could be the bottleneck. To clarify this point, we ran the same experiment with 12 CPUs for the pVM. The results on Fig. 4 bottom show that with enough resources, the performance of the tested application remains almost constant, which proves that resources allocated to the pVM are the bottleneck.

\section{Impact on management tasks}

The pVM hosts VM management operations, the most important ones being: VM creation, destruction and migration. The saturation of the pVM can lead to execution time variation for these operations since they require a significant amount of resources. Fig. 5 left and right respectively show VM creation and migration times according to the pVM load. We observe that the pVM load has a significant impact on these execution times. This situation may dramatically influence cloud services such as auto-scaling [8].

\section{B. $\left(Q_{2}\right)$ pVM location}

On a NUMA machine, the location of the resources allocated to the pVM may significantly influence uVMs performance. The commonly used strategy is to locate all pVM resources on a dedicated NUMA socket, not used by any $\mathrm{uVM}$. This section shows that running the pVM close to uVMs may improve the performance of the latter.

\section{I/O intensive applications' improvement}

We executed a web application in a uVM whose entire CPU and memory resources were located on the same NUMA socket as the pVM. Then, we varied the location of the pVM resources. We observed that the best performance is obtained when the pVM and the uVM share the same NUMA socket (521 req/sec and $8.621 \mathrm{~ms}$ latency if colocated vs $491 \mathrm{req} / \mathrm{sec}$ and $13.431 \mathrm{~ms}$ latency if not). This is because colocation on the same socket prevents remote memory accesses (to fetch $\mathrm{I} / \mathrm{O}$ packets) from both $\mathrm{pVM}$ and $\mathrm{uVM}$ tasks.

\section{VM migration and destruction improvement}

We also observed that running the pVM close to uVMs may improve some management tasks such as live migration. For instance, we observed in our testbed that the migration of a 2GB RAM uVM can be improved by about $34.15 \%$ if the migration process running in the $\mathrm{pVM}$ is scheduled on the same NUMA socket which hosts the migrated uVM's memory. We made a similar observation for uVM destruction tasks: the scrubbing step (memory zeroing) is much faster when the migration process runs close to the NUMA socket which hosts the uVM's memory.

\section{C. $\left(Q_{3}\right)$ pVM resource charging}

The commonly used strategy is to leave the provider support the entire $\mathrm{pVM}$ resources, which includes the resources used on behalf of uVMs for performing I/O operations. [9] showed that this is a vulnerability, which could lead to deny of service attacks.

\section{CONTRIBUTIONS}

\section{A. Design principle}

We introduce Closer (promotes proximity to uVMs), a design principle for implementing a pVM which solves the previously identified issues.

The issues presented in Section III can be summarized by one word : correlation. If the $\mathrm{pVM}$ relies on a standard OS, its resource management does not consider the correlation between pVM tasks and uVMs activities. However, a significant part of pVM tasks are correlated with uVMs activities : (1) in terms of quantity, as the amount of resources required by the $\mathrm{pVM}$ depends on uVMs activities (especially I/O operations), and (2) in terms of location in a NUMA architecture, as resources allocated for one pVM task (e.g. memory) are likely to be used by correlated uVM activities.

To take into account this correlation, our Closer principle influences the design of the pVM with the three following rules:

- on-demand. Resource allocation to the pVM should not be static. Resources should be allocated on demand according to $\mathrm{uVMs}$ activities. Without this rule, resources would be wasted if over-provisioned or they would be lacking, leading to performance unpredictability.

- pay-per-use. The resources allocated to a pVM's task which is correlated with a uVM activity should be charged to the concerned uVM. Without this rule, the $p V M$ would be vulnerable to DoS attacks from a VM executing on the same host, which could use most of the resources from the $\mathrm{pVM}$.

- locality. The resources allocated to a pVM's task which is correlated with a uVM activity should be located as close as possible (i.e. on the same NUMA socket) to the uVM activity. This rule allows reducing remote memory accesses in the NUMA architecture.

To enforce the Closer principle, we introduce an architecture where the pVM is organized in two containers (see Fig. 6): a Main Container (MC) and a Secondary Container (SC). Each 


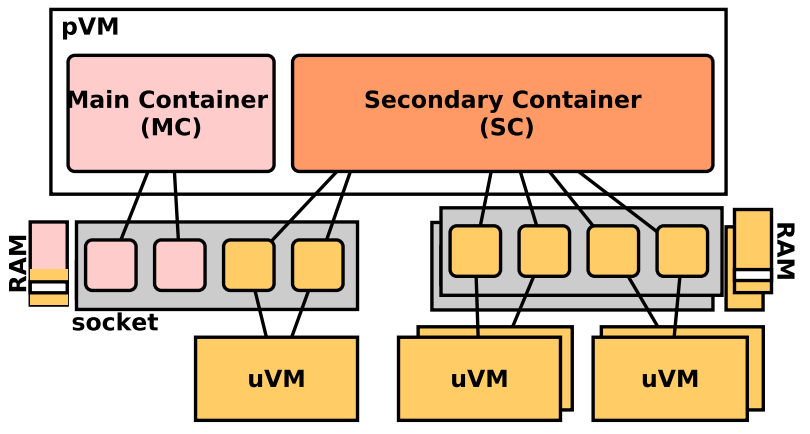

Fig. 6. The new pVM architecture.

container is associated with a specific resource management policy, which controls pVM's resource mapping on physical resources. We implemented this architecture by revisiting Linux instead of building a new pVM from scratch. By this way, we propose a ready to use pVM.

The MC is intended to host tasks (i.e. processes) whose resource consumption is constant (i.e. do not depend on $\mathrm{uVM}$ activities). Other tasks which depend on the activity of a uVM are hosted in the SC. More precisely, pVM tasks are organized into four groups: ( $\left.T_{1}\right)$ OS basic tasks (Linux in our case), $\left(T_{2}\right)$ tasks belonging to the datacenter administration framework (e.g. novaCompute in OpenStack), $\left(T_{3}\right) \mathrm{VM}$ management tasks (create, destroy, migrate, etc.) and $\left(T_{4}\right)$ I/O management tasks (drivers and backends, see Fig. 3). Tasks from $T_{1}$ and $T_{2}$, and almost all tasks from $T_{3}$ (except VM destruction and migration tasks) have a constant resource consumption and are executed in the MC. All other tasks use resources according to $\mathrm{uVMs}$ activities and they are executed in the SC.

\section{B. Resource management}

\section{Description (see Fig. 6)}

At physical machine startup, the pVM is configured with as many vCPUs as available physical processors (called cores). Each vCPU is pinned on a core (one per core). A subset of vCPUs (therefore of cores) of the $\mathrm{pVM}$ is associated with the MC (i.e. used for MC tasks). The rest of vCPUs are linked to the SC and their associated cores are shared with uVMs. Therefore, when a core is reserved for a $\mathrm{uVM}$ (the core is the allocation unit), two vCPUs are pinned on that core: the uVM's vCPU and the SC's vCPU associated with that core. This allows the SC to execute the tasks correlated with the $\mathrm{uVM}$ on its reserved core (therefore to charge the used resources to the $\mathrm{uVM}$ ), following the pay-per-use and locality rules. Regarding the on-demand rule, the $\mathrm{MC}$ is allocated a fixed amount of resources (vCPU and memory) according to its constant load and the $\mathrm{SC}$ is granted a variable amount of resources according to tasks scheduled on its vCPUs.

\section{Resource management for the main container}

The resources allocated to the $\mathrm{MC}$ must be on provider fee, as they are used for executing datacenter administrative tasks (e.g. monitoring). These resources are constant and can be estimated as they only depend on the datacenter administration system (OpenStack, OpenNebula, CloudStack, etc.). Neither the number of VMs nor VMs' activities have an impact on $\mathrm{MC}$ resource consumption. Therefore, we use a static allocation for MC resources at physical machine startup and these resources are located on a reduced number of processor sockets. Through calibration, we can estimate the resource to be allocated to the MC. The evaluation section (Section V) provides such estimations for the most popular cloud administration systems.

\section{Resource management for the secondary container}

The SC includes as many vCPUs as available cores on the physical machine, excluding cores allocated to the MC. At physical machine startup, the SC hosts I/O tasks from the split-driver model (see Fig. 3) for uVMs. Even if the I/O tasks are not active at this stage, they require memory for initialization. This initial memory (noted SCInitialMem²) is very small and assumed by the provider. It will be also used for uVM destruction and migration tasks. Algorithm 1 synthesizes the task scheduling policy in the SC. When a $\mathrm{uVM}$ is at the origin of a task (e.g. for an I/O operation), one of its vCPU is scheduled-out and its associated core is allocated to the SC's vCPU mapped to that core, in order to execute this task. The following sections detail the implementation of this algorithm for $\mathrm{I} / \mathrm{O}$, destruction and migration tasks.

Input : $T$ : an $\mathrm{I} / \mathrm{O}$ task or $\mathrm{VM}$ administration task that should run within $\mathrm{pVM}$

1 targetuVM=Identification of the target $\mathrm{uVM}$

2 targetCPU=Pick a CPU which runs targetuVM

3 Schedule $T$ on the pVM's vCPU which is pinned on targetCPU

Algorithm 1: Task scheduling in the SC.

\section{I/O scheduling in the SC}

pVM's tasks which are executing I/O operations on the account of uVMs are twofold: backends and drivers. The challenge is to identify from the pVM the uVM responsible for each task, in order to use one of its allocated processors for scheduling this task, and to allocate I/O memory buffers as close to that processor as possible. Given that the split-driver structure is both used for network and disk I/O, we describe in the following our solution for networking tasks which are of two types: packet reception and emission from a uVM.

\section{Packet reception}

For illustration, let us consider the Xen implementation (see Fig. 7), although the description below could be applied to other hypervisors which rely on the split-driver model. When

\footnotetext{
${ }^{2}$ The amount of SCInitialMem depends on the number of backend tasks which is bound by the number of vCPU in the SC. We estimated that SCInitialMem accounts for about 10MB per backend instance in Xen as an example.
} 


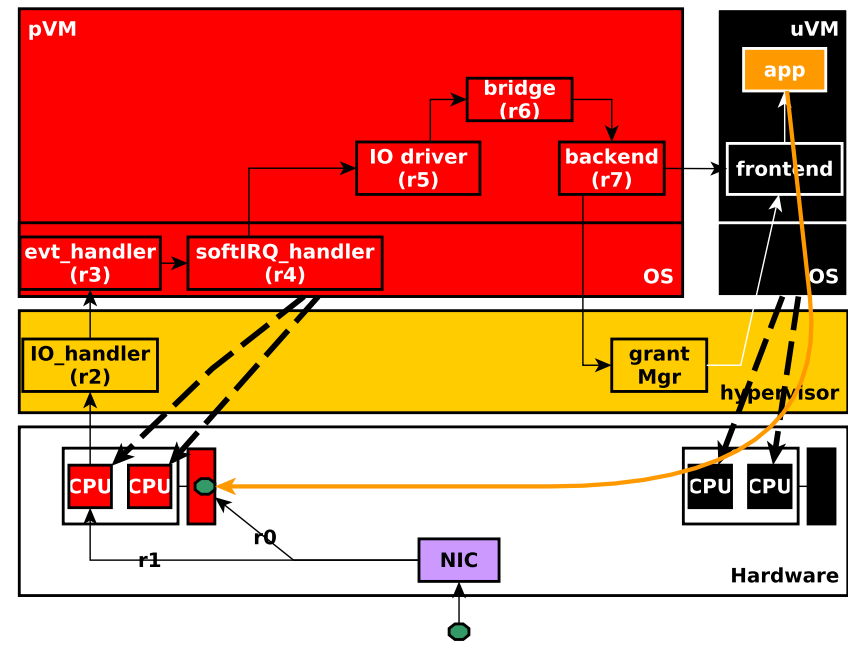

Fig. 7. Packet reception workflow.

the network adapter receives a packet, $\left(r_{0}\right)$ it places the packet in a queue which was initially allocated in main memory and then triggers an interrupt. $\left(r_{1}\right)$ This interrupt is transmitted to one of the processors of the physical machine by the IOAPIC. $\left(r_{2}\right)$ The interrupt handler which lies in the hypervisor notifies to the $\mathrm{pVM}$ the presence of a packet as follows. A vCPU from the pVM (generally vCPU 0 ) is notified (thanks to the event channel mechanism) and is responsible for reacting to this event. The hypervisor then boosts (prioritizes) this vCPU in its scheduler. When the vCPU is scheduled, it detects the presence of an event and executes the event handler $\left(r_{3}\right)$. This handler generates a softIRQ on one of the vCPU from the $\mathrm{pVM}$. The handler of this softIRQ $\left(r_{4}\right)$ triggers the treatment of the packet which will flow up in the network protocols. There are actually two ways of treatment: the traditional treatment which works on a per packet basis (we call it OAPI for old API) and a new one (that we call NAPI for new API) which groups message handling in order to reduce the number of interrupts. $\left(r_{5}\right)$ In both cases, the packet has to be copied from the queue to a skbuff structure (via the copybreak primitive) and the network adapter can then be notified that the packet was well received. The packet is then forwarded to the protocol stack according to the protocol identified in the packet header. In a virtualized system, the destination of the packet is the bridge. $\left(r_{6}\right)$ The bridge identifies from the packet header (which includes a target MAC address) the destination backend. The packet then flows down in the protocol stack to the backend. $\left(r_{7}\right)$ On reception, the backend shares with the destination $\mathrm{uVM}$ the memory pages which include the packet (this is called page flipping) and sends a signal to that $\mathrm{uVM}$. The intervention of the $\mathrm{pVM}$ stops here and the packet continues its path in the $\mathrm{uVM}$ (starting from the frontend).

In order to implement our Closer principle, the general orientation is to force the execution of all $r_{i}$ steps on one of the processors of the target $\mathrm{uVM}$ and to allocate the buffer for the packet on the same socket as that processor.
After step $r_{0}$, the incoming packet has been inserted in a queue in main memory and an interrupt triggered on one processor (generally processor 0 ). The main issue is then to execute the other steps on a processor of the target $\mathrm{uVM}$, while the target $\mathrm{uVM}$ is known only at the level of the bridge (step $r_{6}$ ). Regarding step $r_{1}$, we rely on IRQbalance [15] to balance interrupts between SC's processors (associated with uVMs vCPUs). It does not guarantee that interrupts will be handled by a processor from the target $\mathrm{uVM}$, but it ensures that the MC is not charged for these interrupt handlings, which will be uniformly executed by uVMs processors. This is unfair for $\mathrm{uVMs}$ which execute less I/O operations, but the unfairness is limited to step $r_{1}$ and mitigated as follows. In the hypervisor, we monitor the $\mathrm{I} / \mathrm{O}$ activity of each $\mathrm{uVM}^{3}$ and the notification of a vCPU from the SC (step $r_{2}$ ) is done in proportion to the I/O activity of each $\mathrm{uVM}$ (the $\mathrm{uVM}$ with the highest I/O activity will receive more notifications on its processors, i.e. SC's vCPUs pinned on these processors will be more solicited). This solution (called hyperLB ${ }^{4}$ ) is fair, but not precise and inadequate regarding memory management, as the memory hosting the skbuff buffer will be allocated on the local socket (of the selected processor from the most I/O loaded $\mathrm{uVM}$ ) which could be different from the socket of the final target processor. To prevent such a situation, we perform the identification of the target backend (and therefore of the target $\mathrm{uVM}$ ) in step $r_{4}$ (it was previously performed in step $r_{6}$ in the bridge) and force the execution of the following steps on one of the processors of that $\mathrm{uVM}$. This solution performs well in the OAPI case where each packet reception generates an interrupt and the execution of all the steps for each packet. In the NAPI case, a single interrupt can be generated for the reception of a group of packets (with different target uVMs), whose treatment relies on a poll mechanism. We modified the poll function within the driver (we did it for $e 1000$, this is the only non generic code) in order to identify the target $\mathrm{uVM}$ for each polled packet. In the rest of the document, we call NAPI-1 the per group implementation while NAPI-n refers to the per packet implementation.

\section{Packet emission}

The out-going path is the reverse of the receive path presented in Fig. 7. Applying our Closer principle is in this case straightforward since the $\mathrm{uVM}$ responsible for the I/O activity is known from the beginning step. We simply enforce the hypervisor to notify the vCPU of the SC associated with a processor of the uVM. Then, we modified the pVM's scheduler for the following steps to be executed on the same processor. The same implementation is used for disk operations.

\section{D. $u V M$ destruction and migration scheduling in the $S C$}

$\mathrm{uVM}$ destruction and migration are administration tasks which are also executed in the SC. Logically, resources consumed by these tasks should be charged to the provider.

\footnotetext{
${ }^{3}$ The monitoring is implemented at the level of the interface between the $\mathrm{pVM}$ backend and the uVM frontend.

${ }^{4} \mathrm{LB}$ stands for load balancing.
} 
In our solution, this is effectively the case for memory (with the allocation of SCInitialMem described above), but not for CPU which is charged to the concerned uVM. This is not a problem as the goal is here to remove the uVM from the host, and it has a main advantage: the proximity with the memory of the uVM.

\section{uVM destruction}

The most expensive step in the destruction process of a $\mathrm{uVM}$ is memory scrubbing [8]. This step can be accelerated if the scrubbing process executes close to the uVM memory (following the locality rule). However, the current implementation consists of a unique process which performs that task from any free vCPU from the pVM. Our solution is as follows. Let $S_{i}, i \leq n$ be the sockets where the uVM has at least one vCPU. Let $S_{j}^{\prime}, j \leq m$ be the sockets which host memory from the uVM. For each $S_{i}$, a scrubbing task is started on a vCPU from the $\mathrm{SC}$, the processor associated with this vCPU being local to $S_{i}$ and shared with a vCPU of the uVM. This task scrubs memory locally. The uVM memory hosted in a $S_{j}^{\prime}$ which is not in $S_{i}$ is scrubbed by tasks executing on other sockets (this remote scrubbing is balanced between these tasks).

\section{uVM live migration}

uVM live migration mainly consists in transferring memory to the destination machine. The current implementation consists of a unique process which performs the task from any free vCPU from the pVM. The transfer begins with cold pages. For hot pages, the process progressively transfers pages which were not modified between the previous transfer and the current one. An already transferred page which was modified is transferred again. When the number of modified pages becomes low or the number of iterations equals five, the $\mathrm{uVM}$ is stopped and the remaining pages are transferred. Then, the uVM can resume its execution on the distant machine. We implemented a distributed version of this algorithm which runs one transfer process per socket hosting the uVM's memory (similarly to the destruction solution), thus enhancing locality. These processes are scheduled on SC's vCPUs associated with free processors if available. Otherwise, the processors allocated to the uVM are used.

\section{EVALUATIONS}

This section presents the evaluation results of our revisited Linux and Xen prototype.

\section{A. Experimental setup and methodology}

Servers. We used two Dell servers having the following characteristics: two sockets, each linked to a 65GB memory node; each socket includes 26CPUs $(1.70 \mathrm{GHz})$; the network card is Broadcom Corporation NetXtreme BCM5720, equidistant to the sockets; the SATA disk is also equidistant to the sockets. We used Xen 4.7 and both the pVM and uVMs run Ubuntu Server 16.04 with Linux kernel 4.10.3. Unless otherwise specified, each uVM is configured with four vCPUs,
TABLE I

THE BENCHMARKS WE USED FOR EVALUATIONS.

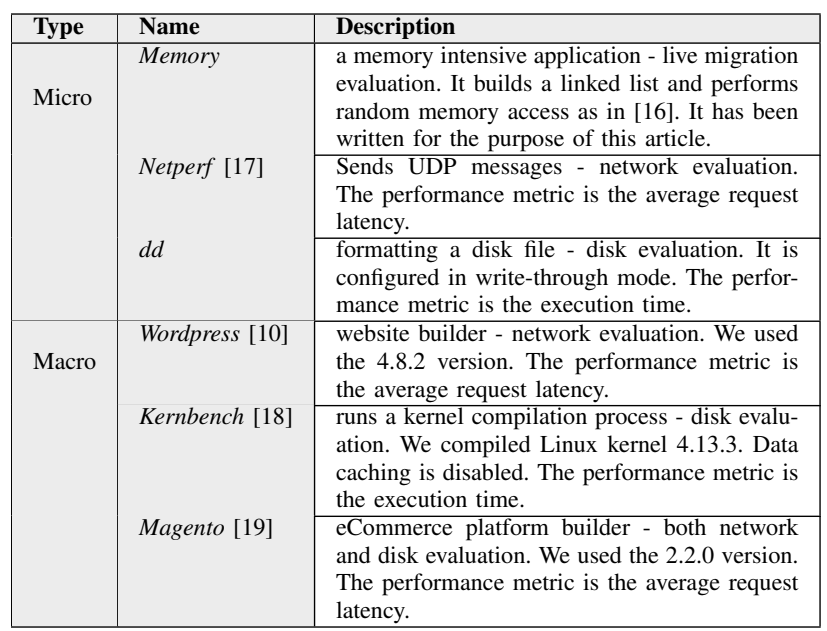

4GB memory and 20GB disk.

Benchmarks. We used both micro- and macro-benchmarks, respectively for analyzing the internal functioning of our solutions and for evaluating how real-life applications are impacted. Table I presents these benchmarks.

Methodology. Recall that the ultimate goal of our proposed $\mathrm{pVM}$ architecture is to respect the three rules of Closer, presented in Section IV-A: on-demand resource allocation (to avoid resource waste and enforce predictability), locality (to improve performance), and pay-per-use (to prevent DoS attacks). As a first step, we separately demonstrate the effectiveness of our architecture for each principle. Notice that the evaluation of a given principle may not include all contributions. Therefore, a final experiment is realized with all the contributions enabled. For each experiment, we compare our solution with the common pVM's resource allocation strategy (referred to as Vanilla pVM, Vanilla for short). In the latter, a set of resources, which are located at the same place, are dedicated to the pVM. Otherwise specified, we dedicate a NUMA socket and its entire memory node to the pVM, corresponding to an oversized allocation.

\section{B. Resource allocation to the $M C$}

In our solution, MC's resources are statically estimated by the provider. We estimated MC's resources for the majority of cloud management systems namely: OpenStack Ocatax [20], OpenNebula 5.2.1 [21] and Eucalyptus 4.4.1 [22]. For each system, we relied on the default configuration and measured its resource consumption. The results, based on our testbed, are presented in Table II. We can see that very few resources are needed for performing all MC's tasks. Our experiments also confirmed that MC's needs do not depend on the IaaS size.

\section{Locality benefits}

We evaluated the benefits of locality on both administrative (uVM destroy and uVM migration) and I/O tasks. Recall that 
TABLE II

MC'S NEEDS FOR THREE CLOUD MANAGEMENT SYSTEMS.

\begin{tabular}{|l|c|c|c|}
\hline & OpenStack & OpenNebula & Eucalyptus \\
\hline \# vCPUs & 2 & 2 & 1 \\
\hline RAM (GB) & 2 & 1.85 & 1.5 \\
\hline
\end{tabular}

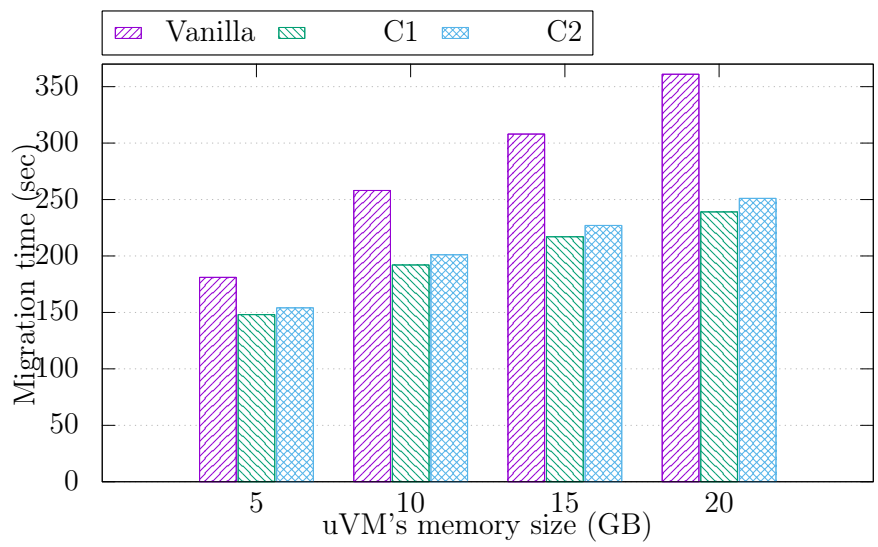

Fig. 8. Multi-threaded migration process (lower is better). The uVM runs a memory intensive application.

we provide locality by enforcing the execution of SC's vCPUs on the same socket as the concerned uVM. In order to only evaluate locality benefits, SC's vCPUs do not use uVM's CPUs (unless otherwise specified).

1) Administrative task improvement: The effectiveness of our uVM destruction solution is obvious and has been well demonstrated in [8] (Subsequently, we do not claim this innovation and we recommend the reader to refer to [8]). We focus on the novel multi-threaded migration solution we propose, whose effectiveness is not obvious due to the management of dirty pages. To this end, we evaluated our solution when the migrated uVM runs an intensive Memory benchmark. We experimented with different uVM memory sizes (4GB-20GB). For every experiment, the uVM's memory is equally spread over the two NUMA nodes of the server. We considered two situations (noted $C_{1}$ and $C_{2}$ ): in $C_{1}$, migration threads do not share the uVM's CPUs (assuming there are free CPUs available on the socket for migration) while they do in $C_{2}$ (assuming there aren't any available free CPU, the socket which runs the uVM being fully occupied). Fig. 8 presents the evaluation results (lower is better). We can see that our solution outperforms Vanilla in all experimented situations. The improvement is most important with large uVMs (up to $33 \%$ in $C_{1}$, see Fig. 8). Intuitively, the reader can imagine that the improvement will also increase when the number of sockets hosting the uVM's memory increases. $C_{1}$ slightly outperforms $C_{2}$ (up to $4 \%$ ), justifying our strategy to only use the uVM's CPUs when there is no free CPU on the involved sockets.

2) I/O task improvement: We evaluated the benefits of running $\mathrm{pVM}$ 's tasks that are involved in a uVM's I/O activity on the same socket as that $\mathrm{uVM}$. We evaluated both network

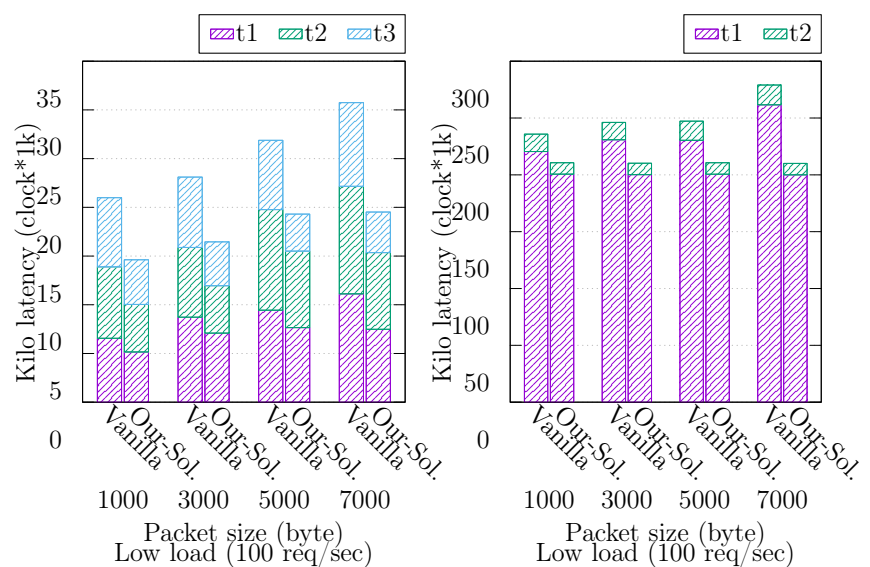

Fig. 9. Packet reception (left) and packet emission (right) improved by locality. (lower is better)

and disk activities. Concerning the former, we evaluated each version presented in Section IV-C, namely: hyperLB at hypervisor level; OAPI, NAPI-1 or NAPI-n at the pVM level. For these evaluations, we used a constant low load (100 req/sec). Packet reception with a single $\mathrm{uVM}$

The uVM is configured with $4 \mathrm{vCPUs}$ (all located on the second socket) and 4GB memory. Another server runs Netperf. We are interested in the following metrics: $\left(t_{1}\right)$ the treatment duration before the backend invocation (this includes $r_{3-6}$ steps, see Fig. 7); $\left(t_{2}\right)$ the time taken by the backend to inform the frontend (step $\left.r_{7}\right)$; and $\left(t_{3}\right)$ the time taken by the frontend to transfer the packet to the application's buffer. Fig. 9 left presents the evaluation results of each $t_{i}$ while varying packet size. Here, all versions provide almost the same values, thus they are shown under the same label (Our-sol.) in Fig. 9 left. We can see that our solution minimizes all $t_{i}$ in comparison with Vanilla, leading to a low $t_{1}+t_{2}+t_{3}$ (up to $36.50 \%$ improvement).

\section{Packet reception with multiple uVMs}

The previous experiments do not show any difference between our implementation versions. The differences appear only when the server runs several uVMs. To this end, we performed the following experiment. The testbed is the same as above in which a second $\mathrm{UVM}$ (noted $d_{2}$, the first one is noted $d_{1}$ ) runs on the first socket (the same which runs the MC). The two uVMs receive the same workload. We are interested in $d_{1}$ 's performance and we compare it with its performance when it runs alone (as in the previous experiments). Fig. 10 left presents $t_{1}+t_{2}+t_{3}$ normalized over Vanilla. We can see that OAPI and NAPI-n outperform NAPI- 1 by about $21 \%$. This is because NAPI-1 (which does a batch treatment) fails in choosing the correct target $\mathrm{uVM}$ for many request in $r_{4}$. This is not the case neither in OAPI nor in NAPI-n which are able to compute the correct destination for each packet. Fig. 10 right presents the amount of errors (wrong destination) observed for each implementation version. Thus, in further experiments, "our solution" refers to OAPI or NAPI-n. 

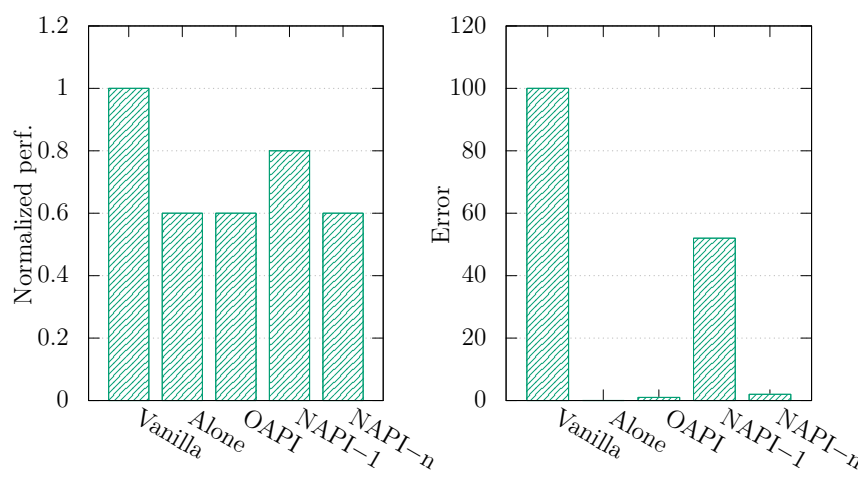

Fig. 10. Packet reception improved by locality: multiple uVMs evaluation. Results are normalized over Vanilla (lower is better).

\section{Packet emission}

The testbed here is the same as in the experiment with packet reception and a single uVM, except the fact that Netperf runs inside the $\mathrm{uVM}$. Remember that packet emission is agnostic about the different implementation versions of our solution (see Section IV-C). We are only interested in $t_{1}$ and $t_{2}\left(t_{3}\right.$ is constant). Fig. 9 right presents the results. We can see that thanks to locality, our solution improves (minimizes) $t_{1}$ by up to $42.33 \%$ for large packets in comparison with Vanilla. Compared with packet reception results, the improvement is more significant here because in the case of packet emission with Vanilla, memory is allocated (on emission) on the $\mathrm{uVM}$ socket and pVM's I/O tasks access the packet remotely. This is not the case with packet reception since the packet enters the machine via the pVM's socket (therefore, packet handling is more important in the $\mathrm{pVM}$ than in the $\mathrm{uVM}$ ).

\section{Disk operations}

The testbed here is the same as above except the fact that the benchmark is $d d$. The uVM's hard disk is configured in write-through mode in order to avoid caching. The collected metric is the execution time. We evaluated different write block sizes. Fig. 11 presents the results. We can see that our solution outperforms Vanilla, especially with large blocks (up to $22 \%$ improvement).

\section{Macro-benchmark improvement}

We also evaluated the benefits of locality on macrobenchmarks. The testbed is the same as above in which the benchmark is replaced by a macro-benchmark. Fig. 12 left presents the results normalized over Vanilla. We can see that all the benchmarks are improved with our solution: about $25 \%$ for wordpress, $21 \%$ for Kernbench, and 30\% for Magento.

\section{Pay-per-use effectiveness}

We validated the effectiveness of our architecture in charging to $\mathrm{uVMs}$ resources consumed by the $\mathrm{pVM}$ on their behalf. This includes demonstrating that MC's resource consumption remains constant regardless uVMs activities. We also evaluated the fairness of our solution, meaning that each $\mathrm{uVM}$ is charged proportionally to its activity. We only present the evaluation results for the packet reception experiment (which is the

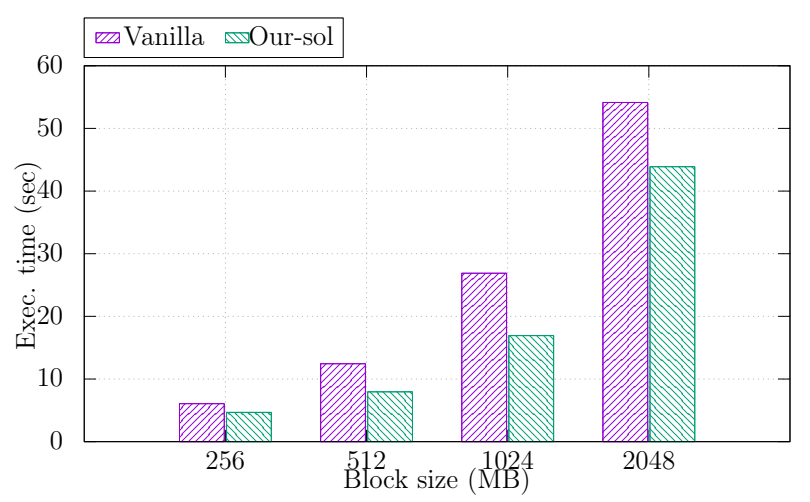

Fig. 11. Disk operations (from $d d$ ) improved by locality. (lower is better).
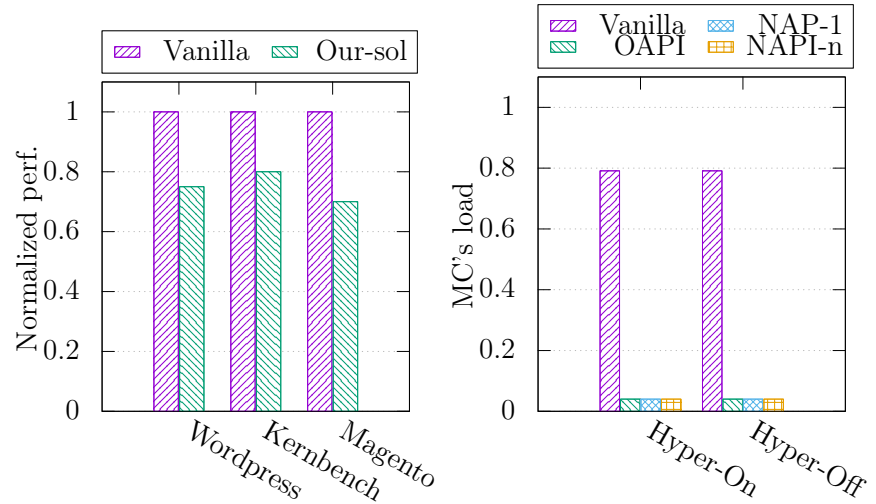

Fig. 12. (left) Macro-benchmarks improved by locality. The results are normalized over Vanilla (the first bar). (right) MC's CPU load.

most sensitive one, packet emission and disk operations being less tricky regarding pay-per-use). To this end, we used the same testbed as previously. The server under test runs three uVMs (noted $v m_{1}, v m_{2}$, and $v m_{3}$ ), each configured with two vCPUs. They share the same socket and each vCPU is pinned to a dedicated CPU. $v m_{1}, v m_{2}$, and $v m_{3}$ respectively receives 1000req/sec (which accounts for $10 \%$ of the total load), 3000req/sec (30\% of the total load) and 6000req/sec (60\% of the total load). In this experiment, we do not care about memory locality. We collected the following metrics: MC's CPU load, and the proportion of CPU time stolen by SC's vCPUs from each uVM (this represents the contribution of the $\mathrm{uVM}$, used for fairness evaluation).

Fig. 12 right shows MC's CPU load consumption while Fig. 13 presents each uVM contribution. In Fig. 12, the ideal solution is the one which leads to no CPU consumption in the $\mathrm{MC}$, meaning that the $\mathrm{MC}$ is not impacted by uVMs activities. The load reported for Vanilla is relative on the MC reserved capacity (e.g. 100\% for Vanilla means that its CPU consumption is equivalent to the entire MC capacity). We can see that all our implementation versions are close to the ideal value, the best version being OAPI/NAPI-1 combined with hyperLB-On while the worst one is NAPI-n 


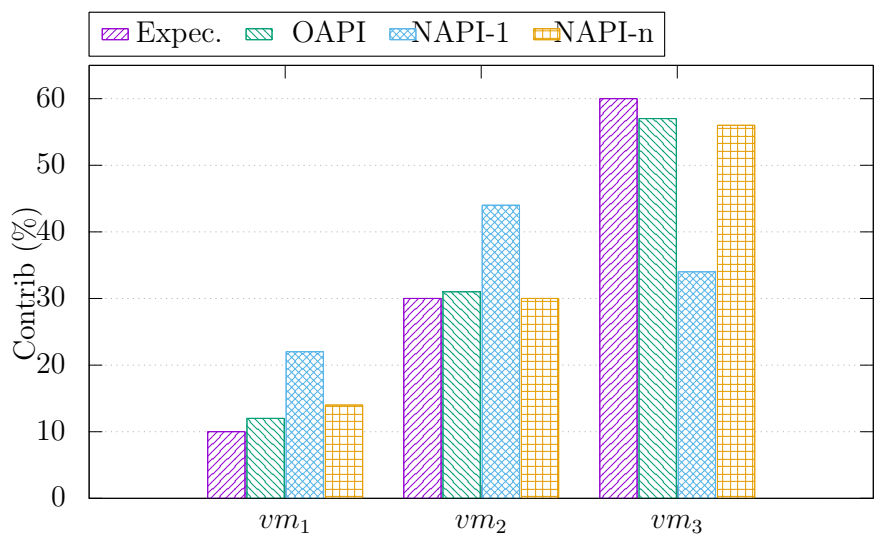

Fig. 13. Fairness of our solutions. (close to the first bar is better).

with hyperLB-Off. But the difference between these versions is very low, meaning that the activation of hyper $L B$ is not necessary. In other words, $r_{3}$ 's CPU consumption is negligible. Therefore, in Fig. 13, we only present results with hyperLB-Off (hyperLB-On provides the same results). The ideal solution is the one which provides the expected result (the first bar). The latter corresponds to the proportion of the uVM's I/O traffic in the total traffic generated by all uVMs. We can see that except NAPI-1, both OAPI and NAPI-n ensure fairness.

\section{E. All together}

We evaluated our solution when locality and pay-per-use mechanisms are enabled at the same time. The testbed is the same as above where macro-benchmarks replace microbenchmarks. We tested all possible colocation scenarios. Our solution is compared with Vanilla when the pVM is allocated the same amount of resources as the MC. Given a benchmark, the baseline value is the one obtained when it runs alone. Having already discussed the impact on performance, the interesting subject here is performance predictability. Several studies [7], [23] showed that this issue may occur when the pVM is saturated due to uVMs activities. We also compared our solution with [7] (called Teabe here). The latter presented a solution which ensures that the aggregated CPU time (including the one generated inside the $\mathrm{pVM}$ ) used by a uVM cannot exceed the capacity booked by its owner. Fig. 14 presents the results for Kernbench using the best implementation version (NAPI-n with HyperLB-Off), all benchmarks running at the same time. In Fig. 14, each evaluation $<$ situation $>$ $<$ solution $>$ is done in a given situation and with a given solution. Situation can be alone or col (for colocation of the 3 benchmarks) and Solution identifies the used solutions (all is our full solution). We can see that Teabe enforces predictability as our solution: in Fig. 14 alone-all is almost equal to col.all; and alone-Teabe is almost equal to col.-Teabe. However, our solution improves application performance thanks to our

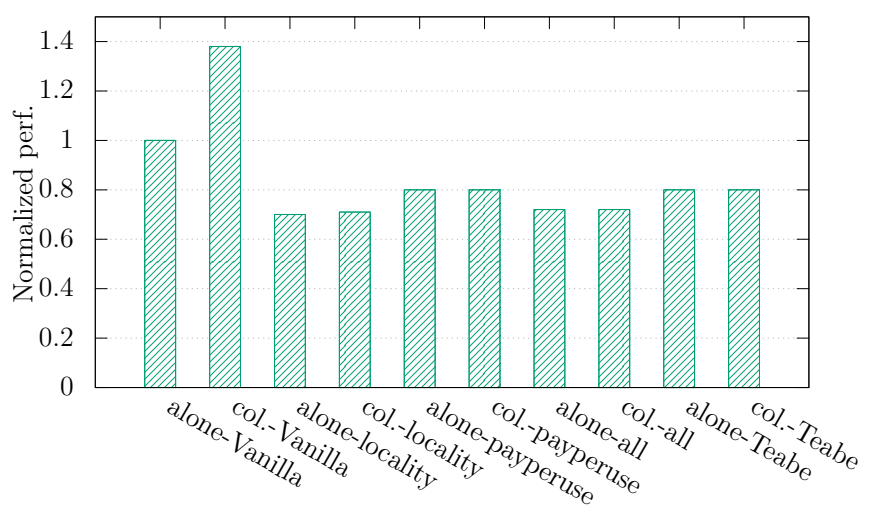

Fig. 14. Our solution with all mechanisms, Kernbench results. The latter are normalized over Vanilla when the benchmark runs alone. For predictability, compare $<$ alone $>-<$ solution $>$ and $<$ col. $>-<$ solution $>$. For performance improvement, lower is better.

locality enforcement contribution: alone-all (respectively col.all) performs better than alone-Teabe (respectively col.-Teabe). As mentioned above, the improvement will increase when the number of sockets hosting the uVMs memory increases. Fig. 14 also shows the results reported in the previous sections so that the reader can easily appreciate on the same curve the impact of each contribution.

\section{RELATED WORK}

Several research studies have investigated pVM management task improvements. Most of them have focused on uVM creation [8], [24]-[32] and migration [33]-[38] in order to provide reactive applications (quick elasticity) and consolidation systems. Very few of them exploit locality to improve these management tasks as we do.

Related to our scrubbing optimization, a Xen patch [39] was proposed to delay the scrubbing process and perform it during idle CPU cycles. Contrarily to our solution in which scrubbing is done synchronously with uVM destruction, [39] has the drawback of letting a completely arbitrary amount of time be spent before the memory is available again, introducing a lot of non-determinism. [8] presents a solution which is quite similar to the one we proposed.

Concerning live migration improvement, we didn't find any solution using a multi-threaded algorithm in order to execute in parallel and close to the migrated uVM's memory as we do.

Regarding I/O virtualization improvement, existing solutions either act at the hypervisor scheduler level [40]-[45] in order to minimize the scheduling quantum length thus minimizing I/O interrupt handling latency.

Very few researchers studied pVM resource utilization (on behalf of uVMs) from the pay-per-use perspective. To the best of our knowledge, [23] and [7] are the only studies which investigated pVM's resource partitioning as we do. [23] is limited to mono-processor machines while [7] leads to resource waste. 


\section{CONCLUSION}

This paper identified several issues related to the design of the pVM in today's virtualization systems. These issues arise from the fact that current pVMs rely on a standard OS (e.g., Linux) whose resource manager does not consider the correlation between pVM's tasks and uVM's activities. These issues lead to resource waste, low performance, performance unpredictability, and vulnerability to DoS attacks.

To take into account this correlation between virtual machines, we introduce Closer, a principle for implementing a suitable OS for the pVM. Closer promotes the proximity and the utilization of $\mathrm{uVMs}$ resources. It influences the design of the pVM with three main rules: on-demand resource allocation, pay-per-use resource charging and locality of allocated resources in a NUMA architecture. We designed a pVM architecture which follows Closer and demonstrated its effectiveness by revisiting Linux and Xen. An intensive evaluation of our implementation using both micro- and macro-benchmarks shows that this architecture improves both management tasks (destruction and migration) and application performance (I/O intensive ones), and enforces predictability.

\section{ACKNOWLEDGMENT}

This work was supported by the HYDDA Project (BPI Grant) and the IDEX IRS (COMUE UGA grant).

\section{REFERENCES}

[1] E. Bugnion, S. Devine, K. Govil, and M. Rosenblum, "Disco: Running commodity operating systems on scalable multiprocessors," ACM Trans. Comput. Syst., vol. 15, no. 4, pp. 412-447, Nov. 1997. [Online]. Available: http://doi.acm.org/10.1145/265924.265930

[2] P. Barham, B. Dragovic, K. Fraser, S. Hand, T. Harris, A. Ho, R. Neugebauer, I. Pratt, and A. Warfield, "Xen and the art of virtualization," in Proceedings of the Nineteenth ACM Symposium on Operating Systems Principles, ser. SOSP '03. Bolton Landing, NY, USA: ACM, 2003, pp. 164-177. [Online]. Available: http://doi.acm.org/10.1145/945445.945462

[3] B. Bui, D. Mvondo, B. Teabe, K. Jiokeng, L. Wapet, A. Tchana, G. Thomas, D. Hagimont, G. Muller, and N. DePalma, "When extended para - virtualization (xpv) meets numa," in Proceedings of the Fourteenth EuroSys Conference 2019, ser. EuroSys '19. Dresden, Germany: ACM, 2019, pp. 7:1-7:15. [Online]. Available: http://doi.acm.org/10.1145/3302424.3303960

[4] vmware.com, "Migration disabled with vmware vsphere sr-iov," Apr. 2018. [Online]. Available: https://goo.gl/d8MGD5

[5] Oracle.com, "Migration disabled with oracle vm server sr-iov," Nov. 2017. [Online]. Available: https://goo.gl/uneSHr

[6] Redhat.com, "Migration disabled with hyper-v sr-iov," Mar. 2015. [Online]. Available: https://goo.gl/qUtsQz

[7] B. Teabe, A. Tchana, and D. Hagimont, "Billing system CPU time on individual VM," in IEEE/ACM 16th International Symposium on Cluster, Cloud and Grid Computing, CCGrid 2016, Cartagena, Colombia, May 16-19, 2016, 2016, pp. 493-496. [Online]. Available: https://doi.org/10.1109/CCGrid.2016.76

[8] V. Nitu, P. Olivier, A. Tchana, D. Chiba, A. Barbalace, D. Hagimont, and B. Ravindran, "Swift birth and quick death: Enabling fast parallel guest boot and destruction in the xen hypervisor," in Proceedings of the 13th ACM SIGPLAN/SIGOPS International Conference on Virtual Execution Environments, ser. VEE '17. Xi' an, China: ACM, 2017, pp. 1-14. [Online]. Available: http://doi.acm.org/10.1145/3050748.3050758

[9] Q. Huang and P. P. Lee, "An experimental study of cascading performance interference in a virtualized environment," SIGMETRICS Perform. Eval. Rev., vol. 40, no. 4, pp. 43-52, Apr. 2013. [Online]. Available: http://doi.acm.org/10.1145/2479942.2479948
[10] Wordpress.org, “Wordpress." Aug. 2012. [Online]. Available: https://wordpress.org/plugins/benchmark/

[11] Pcisig.com, "Sr-iov." [Online]. Available: https://pcisig.com/specifications/iov/

[12] Oracle.com, "Changing the dom0 memory size," Jun. 2014. [Online]. Available: https://docs.oracle.com/cd/E27300_01/E27308/html/vmiugserver-dom0-memory.html

[13] C. Delimitrou and C. Kozyrakis, "Hcloud: Resource-efficient provisioning in shared cloud systems," in Proceedings of the Twenty-First International Conference on Architectural Support for Programming Languages and Operating Systems, ser. ASPLOS '16. Atlanta, Georgia, USA: ACM, 2016, pp. 473-488. [Online]. Available: http://doi.acm.org/10.1145/2872362.2872365

[14] Microsoft., "Microsoft's top 10 business practices for environmentally sustainable data centers 2010," Aug. 2009. [Online]. Available: http://tiny.cc/vro25y

[15] linux.die.net, "Irq balance," Jun. 2013. [Online]. Available: https://linux.die.net/man/1/irqbalance.

[16] U. Drepper, "What every programmer should know about memory," 2007.

[17] HewlettPackard, “Netperf," Jun. 2008. [Online]. Available: https://github.com/HewlettPackard/netperf

[18] kolivas.org, "Kernbench." Apr. 2014. [Online]. Available: http://ck.kolivas.org/apps/kernbench/kernbench-0.50/

[19] magento.com, "Magento," Mar. 2008. [Online]. Available: https://magento.com/

[20] openstack.org, “Openstack," Aug. 2017. [Online]. Available: https://www.openstack.org/

[21] openNebula, "Opennebula," Jul. 2017. [Online]. Available: https://opennebula.org/

[22] D. Nurmi, R. Wolski, C. Grzegorczyk, G. Obertelli, S. Soman, L. Youseff, and D. Zagorodnov, "The eucalyptus open-source cloud-computing system," in Proceedings of the 2009 9th IEEE/ACM International Symposium on Cluster Computing and the Grid, ser. CCGRID '09. Washington, DC, USA: IEEE Computer Society, 2009, pp. 124-131. [Online]. Available: http://dx.doi.org/10.1109/CCGRID.2009.93

[23] D. Gupta, L. Cherkasova, R. Gardner, and A. Vahdat, "Enforcing performance isolation across virtual machines in xen," in Proceedings of the ACM/IFIP/USENIX 2006 International Conference on Middleware, ser. Middleware '06. Melbourne, Australia: SpringerVerlag New York, Inc., 2006, pp. 342-362. [Online]. Available http://dl.acm.org/citation.cfm?id=1515984.1516011

[24] H. A. Lagar-Cavilla, J. A. Whitney, A. M. Scannell, P. Patchin, S. M. Rumble, E. de Lara, M. Brudno, and M. Satyanarayanan, "Snowflock: Rapid virtual machine cloning for cloud computing," in Proceedings of the 4th ACM European Conference on Computer Systems, ser. EuroSys '09. Nuremberg, Germany: ACM, 2009, pp. 1-12. [Online]. Available: http://doi.acm.org/10.1145/1519065.1519067

[25] J. Zhu, Z. Jiang, and Z. Xiao, "Twinkle: A fast resource provisioning mechanism for internet services," in 2011 Proceedings IEEE INFOCOM, April 2011, pp. 802-810.

[26] I. Zhang, A. Garthwaite, Y. Baskakov, and K. C. Barr, "Fast restore of checkpointed memory using working set estimation," in Proceedings of the 7th ACM SIGPLAN/SIGOPS International Conference on Virtual Execution Environments, ser. VEE '11. Newport Beach, California, USA: ACM, 2011, pp. 87-98. [Online]. Available: http://doi.acm.org/10.1145/1952682.1952695

[27] I. Zhang, T. Denniston, Y. Baskakov, and A. Garthwaite, "Optimizing vm checkpointing for restore performance in vmware esxi," in Proceedings of the 2013 USENIX Conference on Annual Technical Conference, ser. USENIX ATC'13. San Jose, CA: USENIX Association, 2013, pp. 1-12. [Online]. Available: http://dl.acm.org/citation.cfm?id=2535461.2535463

[28] T. Knauth and C. Fetzer, "Dreamserver: Truly on-demand cloud services," in Proceedings of International Conference on Systems and Storage, ser. SYSTOR 2014. Haifa, Israel: ACM, 2014, pp. 9:1-9:11. [Online]. Available: http://doi.acm.org/10.1145/2611354.2611362

[29] T. Knauth, P. Kiruvale, M. Hiltunen, and C. Fetzer, "Sloth: Sdn-enabled activity-based virtual machine deployment," in Proceedings of the Third Workshop on Hot Topics in Software Defined Networking, ser. HotSDN '14. Chicago, Illinois, USA: ACM, 2014, pp. 205-206. [Online]. Available: http://doi.acm.org/10.1145/2620728.2620765

[30] A. Madhavapeddy, T. Leonard, M. Skjegstad, T. Gazagnaire, D. Sheets, D. Scott, R. Mortier, A. Chaudhry, B. Singh, 
J. Ludlam, J. Crowcroft, and I. Leslie, "Jitsu: Just-in-time summoning of unikernels," in 12th USENIX Symposium on Networked Systems Design and Implementation (NSDI 15). Oakland, CA: USENIX Association, 2015, pp. 559-573. [Online]. Available: https://www.usenix.org/conference/nsdi15/technicalsessions/presentation/madhavapeddy

[31] D. E. Porter, S. Boyd-Wickizer, J. Howell, R. Olinsky, and G. C. Hunt, "Rethinking the library os from the top down," in Proceedings of the Sixteenth International Conference on Architectural Support for Programming Languages and Operating Systems, ser. ASPLOS XVI. Newport Beach, California, USA: ACM, 2011, pp. 291-304. [Online]. Available: http://doi.acm.org/10.1145/1950365.1950399

[32] F. Manco, C. Lupu, F. Schmidt, J. Mendes, S. Kuenzer, S. Sati, K. Yasukata, C. Raiciu, and F. Huici, "My vm is lighter (and safer) than your container," in Proceedings of the 26th Symposium on Operating Systems Principles, ser. SOSP '17, 2017.

[33] H. Jin, L. Deng, S. Wu, X. Shi, and X. Pan, "Live virtual machine migration with adaptive, memory compression," in 2009 IEEE International Conference on Cluster Computing and Workshops, Aug 2009, pp. $1-10$.

[34] S. Nathan, P. Kulkarni, and U. Bellur, "Resource availability based performance benchmarking of virtual machine migrations," in Proceedings of the 4th ACM/SPEC International Conference on Performance Engineering, ser. ICPE '13. Prague, Czech Republic: ACM, 2013, pp. 387-398. [Online]. Available: http://doi.acm.org/10.1145/2479871.2479932

[35] P. Svärd, B. Hudzia, J. Tordsson, and E. Elmroth, "Evaluation of delta compression techniques for efficient live migration of large virtual machines," in Proceedings of the 7th ACM SIGPLAN/SIGOPS International Conference on Virtual Execution Environments, ser. VEE '11. Newport Beach, California, USA: ACM, 2011, pp. 111-120. [Online]. Available: http://doi.acm.org/10.1145/1952682.1952698

[36] T. Wood, K. K. Ramakrishnan, P. Shenoy, and J. van der Merwe, "Cloudnet: Dynamic pooling of cloud resources by live wan migration of virtual machines," in Proceedings of the 7th ACM SIGPLAN/SIGOPS International Conference on Virtual Execution Environments, ser. VEE '11. Newport Beach, California, USA: ACM, 2011, pp. 121-132. [Online]. Available: http://doi.acm.org/10.1145/1952682.1952699

[37] Y. Abe, R. Geambasu, K. Joshi, and M. Satyanarayanan, "Urgent virtual machine eviction with enlightened post-copy," in Proceedings of the12th ACM SIGPLAN/SIGOPS International Conference on Virtual Execution Environments, ser. VEE '16. Atlanta, Georgia, USA: ACM, 2016, pp. 51-64. [Online]. Available: http://doi.acm.org/10.1145/2892242.2892252

[38] K. Kourai and H. Ooba, "Vmbeam: Zero-copy migration of virtual machines for virtual iaas clouds," in 35th IEEE Symposium on Reliable Distributed Systems, SRDS 2016, Budapest, Hungary, September 26-29, 2016, 2016, pp. 121-126. [Online]. Available: https://doi.org/10.1109/SRDS.2016.024

[39] Xen.org, "xen: free_domheap_pages: delay page scrub to idle loop." May 2014. [Online]. Available: https://lists.xenproject.org/archives/html/xen-devel/201405/msg02436.htm

[40] C. Xu, S. Gamage, P. N. Rao, A. Kangarlou, R. R. Kompella, and $\mathrm{D}$. $\mathrm{Xu}$, "vslicer: Latency-aware virtual machine scheduling via differentiated-frequency cpu slicing," in Proceedings of the 21st International Symposium on High-Performance Parallel and Distributed Computing, ser. HPDC '12. Delft, The Netherlands: ACM, 2012, pp. 3-14. [Online]. Available: http://doi.acm.org/10.1145/2287076.2287080

[41] B. Teabe, A. Tchana, and D. Hagimont, "Application-specific quantum for multi-core platform scheduler," in Proceedings of the Eleventh European Conference on Computer Systems, ser. EuroSys '16. London, United Kingdom: ACM, 2016, pp. 3:1-3:14. [Online]. Available: http://doi.acm.org/10.1145/2901318.2901340

[42] C. Xu, S. Gamage, H. Lu, R. Kompella, and D. Xu, "vturbo: Accelerating virtual machine i/o processing using designated turbo-sliced core," in Presented as part of the 2013 USENIX Annual Technical Conference (USENIX ATC 13). San Jose, CA: USENIX, 2013, pp. 243-254. [Online]. Available: https://www.usenix.org/conference/atc13/technicalsessions/presentation/xu

[43] D. Ongaro, A. L. Cox, and S. Rixner, "Scheduling i/o in virtual machine monitors," in Proceedings of the Fourth ACM SIGPLAN/SIGOPS International Conference on Virtual Execution Environments, ser. VEE
'08. Seattle, WA, USA: ACM, 2008, pp. 1-10. [Online]. Available: http://doi.acm.org/10.1145/1346256.1346258

[44] L. Zeng, Y. Wang, D. Feng, and K. B. Kent, "Xcollopts: A novel improvement of network virtualizations in xen for i/o-latency sensitive applications on multicores," IEEE Transactions on Network and Service Management, vol. 12, no. 2, pp. 163-175, June 2015.

[45] D. Abramson, J. Jackson, S. Muthrasanallur, G. Neiger, G. Regnier, R. Sankaran, I. Schoinas, R. Uhlig, B. Vembu, and J. Wiegert, "Intel virtualization technology for directed i/o," Intel Technology Journal, vol. 10, no. 03, pp. 179-192, August 2006. 